\title{
Diversidad y abundancia de los equinodermos y su relación con la cobertura bentónica del arrecife Enmedio, Sistema Arrecifal Lobos-Tuxpan, México
}

\section{Diversity and abundance of echinoderms and their relation with the benthic cover of the Enmedio Reef, Lobos-Tuxpan Reef System, Mexico}

\author{
Itzel Morales-Quijano ${ }^{1}$, Vicencio de la Cruz-Francisco ${ }^{1 *}$, Marlene González-González ${ }^{l}$ y Jimmy \\ Argüelles-Jiménez ${ }^{2}$
}

\begin{abstract}
RESUMEN
Hasta la fecha, en el Área Natural Protegida, Sistema Arrecifal Lobos-Tuxpan, aún se desconoce la ecología comunitaria de los equinodermos. Por ello, fueron contabilizadas especies de equinodermos en el arrecife Enmedio, utilizando cuadrantes de $1 \mathrm{~m}^{2}$. Los cuadrantes fueron colocados a cada $5 \mathrm{~m}$ sobre tres transectos de $50 \mathrm{~m}$ de largo en el arrecife posterior, laguna arrecifal y arrecife frontal. Con los datos obtenidos se estimaron riqueza de especies, abundancia, densidad, equitatividad, dominancia de Simpson y diversidad verdadera. Asimismo, se determinaron similitudes entre las zonas de muestreo mediante análisis de similitud y ordenación. También se realizó un análisis de correspondencia canónica utilizando como variable ambiental la cobertura bentónica. Se cuantificó un total de 5569 equinodermos, identificándose cinco clases, 10 órdenes, 16 familias, 19 géneros y 23 especies. La densidad de equinodermos en el arrecife fue de $11.23 \mathrm{ind} / \mathrm{m}^{2}$; las especies más importantes en abundancia y densidad fueron: Echinometra viridis (3 389 organismos; $6.85 \mathrm{ind} / \mathrm{m}^{2}$ ) y Echinometra lucunter lucunter (1 803 organismos; $3.64 \mathrm{ind} / \mathrm{m}^{2}$ ). En la laguna arrecifal se registró mayor riqueza, abundancia y densidad de equinodermos, en comparación con las zonas profundas. Los tres ambientes arrecifales mostraron altos valores de dominancia y bajos valores de diversidad verdadera. Las zonas de muestreo presentaron baja similitud y no manifestaron una zonación distintiva entre ambientes arrecifales. Solamente diez especies de equinodermos tuvieron afinidades con la cobertura de corales, macroalgas, algas filamentosas y escombros, estos sustratos parecen ser factores claves en la distribución y abundancia de los equinodermos en el arrecife Enmedio.
\end{abstract}

Palabras claves: Hábitats bentónicos, comunidad bentónica, equinoideos, ofiuras, asteroideos.

\begin{abstract}
To date, the echinoderm community ecology of the Lobos-Tuxpan Reef System's Natural Protected Area is still unknown. For this reason, the echinoderm species in the Enmedio Reef were counted using $1 \mathrm{~m}^{2}$ quadrants placed every $5 \mathrm{~m}$ over three $50 \mathrm{~m}$ long transects in the posterior reef, the reef lagoon and the frontal reef. The species richness, abundance, density, equitability, Simpson's dominance and true diversity were estimated using the data obtained. In addition, similarities were determined between the sampling areas by similarity and ordering analysis. A canonical correspondence analysis was also conducted using benthic cover as environmental variable. A total of 5569 echinoderms were quantified, identifying five classes, 10 orders, 16 families, 19 genera and 23 species. The density of echinoderms on the reef was $11.23 \mathrm{ind} / \mathrm{m}^{2}$;
\end{abstract}

\footnotetext{
1 Facultad de Ciencias Biológicas y Agropecuarias, Campus Tuxpan, Universidad Veracruzana. Carr. Tuxpan-Tampico km 7.5, Col. Universitaria CP. 92860, Tuxpan, Veracruz, México.

2 Instituto de Ciencias Marinas y Pesquerías, Universidad Veracruzana, Hidalgo 617, Col. Río Jamapa, C.P. 94290 , Boca del Río,Veracruz, México. quijanobm93@hotmail.com, delacruzf17@hotmail.com*; marleneglez1392@ gmail.com; cayix24@gmail.com
}

Recibido: 4 de agosto de 2017

Corregido: 6 de noviembre de 2017

Aceptado: 6 de noviembre de 2017

DOI: http://dx.doi.org/10.15359/revmar.9-2.3 
the most important species in terms of abundance and density were Echinometra viridis (3389 organisms, $\left.6.85 \mathrm{ind} / \mathrm{m}^{2}\right)$ and Echinometra lucunter lucunter $\left(1803\right.$ organisms, $\left.3.64 \mathrm{ind} / \mathrm{m}^{2}\right)$. In the reef lagoon a greater richness, abundance and density of echinoderms was recorded, compared to the deep zones. In the three reef environments, high values of dominance and low values of true diversity were recorded. The sampling areas presented low similarity and did not show a distinctive zonation between reef environments. Only ten species of echinoderms showed affinities with corals, macroalgae, filamentous algae and debris; these substrata seem to be key factors in the distribution and abundance of echinoderms in the Enmedio reef.

Keyworks: Benthic habitats, benthic community, echinoids, ophiura, starfish.

\section{INTRODUCCIÓN}

México concentra el 10\% (643 especies) de la diversidad total de equinodermos del mundo, siendo el Golfo de México la región marina más diversa. Se encuentran en este país, 25 especies de crinoideos, 96 de asteroideos, 79 de ofiuras, 59 de equinoideos, 33 de holotúridos, además de la ofiura endémica: Amphiodia guillermosoberoni (SolísMarín et al. 2013). Sin embargo, esta región marina es una de las menos estudiadas con un abordaje ecológico (Solís-Marín et al. 2013), hasta la fecha solamente existen seis publicaciones que analizan la ecología de las comunidades de equinodermos.

El primer trabajo ecológico abordó el análisis cualitativo y cuantitativo de los equinodermos de la laguna de Términos, Campeche, sureste del Golfo de México (Caso, 1994). Otros trabajos se enfocaron en describir aspectos comunitarios y poblacionales básicos (e.g. abundancia, densidad y distribución) como el análisis a distintos intervalos de profundidad (Vázquez-Bader et al. 2008), la distribución y preferencia por el sustrato (Celaya-Hernández et al. 2008), así como la comparación entre la estructura comunitaria y la diversidad morfológica en distintas localidades (González-Azcárraga, 2009). Recientemente, los estudios realizados en el Sistema Arrecifal Veracruzano (SAV) y en el del norte y sur de Veracruz han abordado la abundancia y densidad de los equinodermos en arrecifes coralinos (Pérez-España et al. 2015), así como su distribución y semejanza entre los sistemas de arrecifes (GonzálezGándara et al. 2015).

Si bien, las citadas contribuciones científicas son importantes para las costas mexicanas del Golfo de México, se ha recomendado que es necesario generar más información sobre la abundancia y densidad de los equinodermos y su relación con ambientes bióticos y abióticos (incluyendo datos poblacionales como crecimiento, reproducción y alimentación) (Solís-Marín et al. 2013) en especial en ecosistemas poco explorados (Solís-Marín et al. 2014).

Particularmente en la región norte de Veracruz, solo se han generado listas faunísticas de equinodermos para el arrecife Blake y los arrecifes Lobos, Medio y Blanquilla (GonzálezGándara et al. 2015), por lo que aún se desconocen la composición de especies y el estado actual de las poblaciones 
de equinodermos para el resto de los arrecifes que componen el Sistema Arrecifal Lobos-Tuxpan (SALT). Por ello, los objetivos del presente trabajo fueron aportar los primeros datos de la riqueza, abundancia y densidad de los equinodermos del arrecife Enmedio y establecer si estos descriptores varían entre ambientes arrecifales (laguna arrecifal, arrecife frontal y arrecife posterior), tal y como se ha reportado para los arrecifes del polígono Lobos (González-Gándara et al. 2015) y para otros grupos taxonómicos: esponjas (De la Cruz-Francisco et al. 2016b) y corales (De la Cruz-Francisco et al. 2016c; González-González et al. 2016). Considerando que los equinodermos se distribuyen con base en las preferencias de alimentación y por características del sustrato (Celaya-Hernández et al. 2008; Bologna et al. 2012), en este estudio también se averiguó si la abundancia de las especies de equinodermos se correlaciona con la cobertura de los hábitats bentónicos dominantes que caracterizan al arrecife Enmedio (González-González et al. 2016).

\section{MATERIALES Y MÉTODOS}

Área de estudio: El arrecife Enmedio es uno de los arrecifes de plataforma más pequeños del SALT, con una longitud de $1.06 \mathrm{~km}$ y anchura máxima de $0.48 \mathrm{~km}$ (Tunnell, 2010; SEMARNAT \& CONANP, 2014), se localiza a $14 \mathrm{~km}$ de la desembocadura del Río Tuxpan colindando al norte con el arrecife Tanhuijo y al suroeste con el arrecife Pantepec. Se distinguen claramente tres ambientes arrecifales: arrecife posterior, laguna arrecifal y arrecife frontal (Hill \& Wilkinson, 2004), conocidos también como sotavento, laguna arrecifal y barlovento, respectivamente (Chávez et al. 2010). La laguna arrecifal tiene una profundidad variable que alcanza hasta $2 \mathrm{~m}$ y se han reconocido ocho hábitats bentónicos dominantes constituidos por: Acropora palmata, Millepora alcicornis, Orbicella annularis, Pseudodiploria clivosa, Porites furcata, Palythoa caribaeorum, Eunicea flexuosa y dos componentes bentónicos: cresta arrecifal y escombros (De la Cruz-Francisco et al. 2016a; González-González et al. 2016). El arrecife frontal o barlovento (lado este del arrecife) presenta una pendiente suave y predomina el pavimento coralino. En el arrecife posterior o sotavento (lado oeste del arrecife) dominan colonias de corales masivos representados principalmente por: O. annularis, Colpophyllia natans y Siderastrea siderea (GonzálezGonzález et al. 2016).

La riqueza de especies y la abundancia de equinodermos se obtuvieron a la par con los muestreos realizados por González-González et al. (2016), quienes estimaron la cobertura bentónica en la laguna arrecifal, en el arrecife frontal y en el arrecife posterior. El diseño de muestreo consistió en colocar un cuadrante de $1 \mathrm{~m}^{2}$ a cada $5 \mathrm{~m}$ sobre tres transectos de $50 \mathrm{~m}$ de longitud. En total se abarcó un área de $33 \mathrm{~m}^{2}$ por sitio de muestreo. En las 
pendientes profundas del arrecife, los transectos fueron colocados en forma perpendicular, con el fin de abarcar un mayor rango de profundidad. En el arrecife posterior, los transectos se colocaron a una profundidad de 5 $\mathrm{m}$ y finalizaron aproximadamente a los $15 \mathrm{~m}$ de profundidad, dado que la pendiente es más abrupta en esta zona. En el arrecife frontal, los transectos se tendieron a una profundidad de $5 \mathrm{~m} \mathrm{y}$ finalizaron aproximadamente a los 12 $\mathrm{m}$ de profundidad. Con respecto a la planicie, los transectos se tendieron paralelos al arrecife, a una profundidad de 1-3 m (Fig. 1).
Las especies de equinodermos se identificaron en el campo considerando las características morfológicas y los patrones de coloración que se describen en guías de identificación (Humann \& Deloach, 2002) y catálogos de equinodermos (BenavidesSerrato et al. 2011; Borrero-Pérez et al. 2012). La abundancia de los equinodermos consistió en registrar por especie la cantidad de individuos observados dentro del cuadrante de $1 \mathrm{~m}^{2}$. Mientras la densidad de cada especie se obtuvo dividiendo el total de organismos de cada especie entre el número de cuadrantes realizados

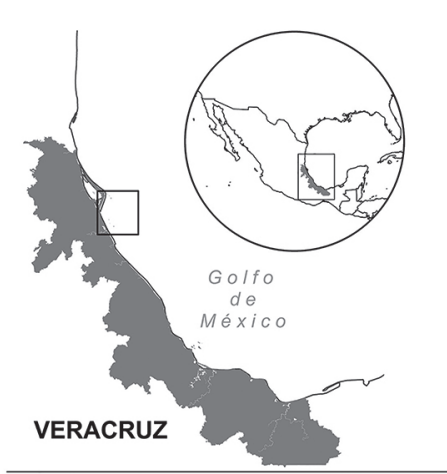

SISTEMA ARRECIFAL LOBOS-TUXPAN

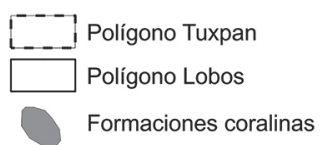

ZONAS DE MUESTREO

Laguna arrecifal $(P)$

Arrecife posterior/sotavento (S)

Arrecife frontal/barlovento $(B)$

Sitios de muestreo: 1, 2, 3, 4, 5

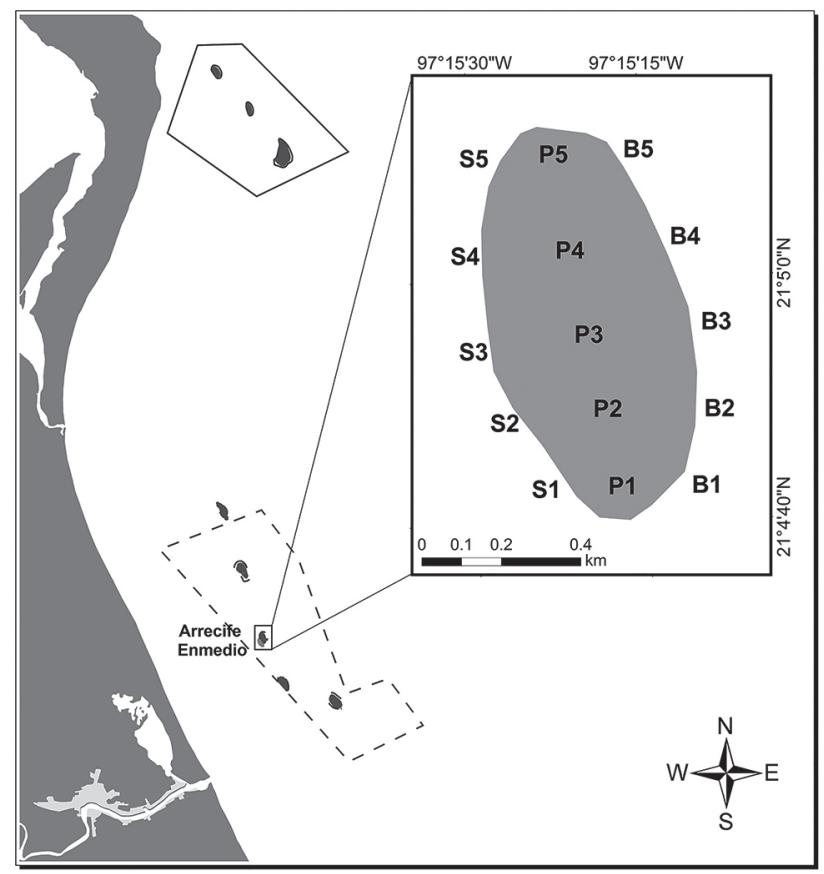

Fig. 1. Localización geográfica del arrecife Enmedio. Se muestra la distribución de los sitios de muestreo en las zonas arrecifales: arrecife posterior, laguna arrecifal y arrecife frontal Fig. 1. Geographical location of the Enmedio reef. The distribution of sampling sites is shown in the reef areas: back reef, reef lagoon and front reef 
por sitio. Dentro del cuadrante se revisaron oquedades de rocas, frondas de macroalgas e intersticios de esponjas y escombros para hallar pequeños equinodermos crípticos como asteroideos, ofiuras y pepinos. Las especies que resultaron dudosas fueron recolectadas e identificadas en el laboratorio, utilizando referencias bibliográficas (Hernández-Herrejón et al. 2008 y Benavides-Serrato et al. 2011; Borrero-Pérez et al. 2012). Todo el material identificado se depositó en la colección de la Facultad de Ciencias Biológicas y Agropecuarias, Universidad Veracruzana.

La eficiencia de los muestreos se evaluó a través del estimador no paramétrico Chao 1. Para tal fin se calculó la representatividad porcentual (\%) del muestreo, dividiendo el valor de la riqueza observada (multiplicado por 100) entre el valor de la riqueza esperada del estimador no paramétrico. El desempeño del estimador Chao $1 \mathrm{se}$ evaluó calculando el sesgo (atributo que indica sobrestimación o subestimación de la riqueza verdadera) y la exactitud (indica la cercanía de la riqueza esperada a la riqueza verdadera) con base en las fórmulas propuestas por Walther \& Moore (2005): Sesgo $=($ Sestimada $)-($ Sverdadera $) /$ Sveradera; Exactitud $=(($ Sestimada $)-$ (Sverdadera)/Sveradera)^2. En ambas fórmulas se obtienen valores entre -1 y 1 , cuando los valores son cercanos a cero indican que el estimador presenta menos sesgo o mayor exactitud, respectivamente. Los valores de la riqueza observada y estimada se obtuvieron con el programa EstimateS, versión 9 (Colwell, 2013) y se graficaron para representar las curvas acumulativas de especies observadas y estimadas.

Asimismo, se utilizó como estimador asintótico el modelo de Clench para predecir el número de especies existentes en cada zona arrecifal calculando la asíntota como la relación $\mathrm{a} / \mathrm{b}$, con este modelo se evaluó la calidad del inventario calculando la pendiente al final de la curva, $a /(1+b \cdot n)^{\wedge} 2$, cuando el valor de la pendiente es menor de 0.1 indica que el inventario es aceptable; también se evaluó la proporción de la riqueza registrada, Sobs/(a/b) y se estimó el esfuerzo de muestreo para alcanzar el $95 \%$ de la proporción de la fauna, N_q=q/[b(1-q)]; todo este proceso se realizó con base en el trabajo de Jiménez-Valverde \& Hortal (2003) y utilizando el programa Statistica 7.1 (StatSoft, 2005), las curvas de especies esperadas se graficaron junto con los estimadores no paramétricos para fines comparativos.

La abundancia y la densidad de las especies de equinodermos se calcularon para el arrecife y por zona arrecifal. Con la información obtenida se estimaron índices basados en la abundancia: riqueza específica, diversidad de Shannon, equitatividad, dominancia de Simpson (D) y diversidad verdadera de orden $1\left({ }^{1} D\right)$, cada atributo comunitario fue estimado para los sitios de muestreo utilizando el programa Primer V7 (Clarke \& Gorley, 2015). La diversidad 
verdadera $\left({ }^{1} \mathrm{D}\right)$ se obtuvo calculando el exponente de la diversidad de Shannon: ${ }^{1} \mathrm{D}=\exp H^{\prime}$ (Jost, 2006), este índice expresa el número efectivo de especies que tendría una comunidad integrada por especies igualmente abundantes, dicho índice tiene la ventaja de realizar comparaciones de la diversidad verdadera de dos o más comunidades (Moreno et al. 2011). Para cada atributo comunitario se determinaron diferencias significativas entre los sitios de muestreo de cada zona arrecifal mediante análisis de varianza (ANOVA) utilizando el programa Statistica 7.1 (StatSoft, 2005).

Para los sitios de muestreo se determinaron semejanzas con base en la riqueza y abundancia de equinodermos, como medida de similaridad se utilizó la distancia de Bray-Curtis, método basado en datos cuantitativos, a partir de la matriz de similitud se generó un dendrograma, con el fin de mostrar el grado de semejanza entre las unidades de muestreo (sitios). Como análisis complementario se utilizó el método de ordenación multidimensional no métrico (nMDS) basándose en la matriz de similitud de Bray-Curtis para representar las proximidades de los sitios de muestreo, además se realizó un análisis de similitud porcentual (SIMPER) para determinar qué especies contribuyen a la diferenciación/ similitud de los grupos formados, dichas técnicas multivariantes se realizaron con el programa Primer, Versión 7 (Clarke \& Gorley, 2015).

Para determinar si las especies de equinodermos muestran alguna relación con la cobertura bentónica del arrecife Enmedio, se realizó un Análisis de Correspondencia Canónica (ACC) debido a que la longitud del gradiente detectado a través de un DCA (Detrended Correspondence Analysis) fue mayor a 0.4 , esto indica una relación unimodal propia de ser analizada por un ACC (Ter Braak \& Smilauer, 2003). Las variables ambientales expresadas en coberturas se tomaron de GonzálezGonzález et al. (2016), siendo estas: algas, esponjas, corales, anémonas, zoántidos, también se consideraron los componentes bentónicos: pavimento coralino (superficie dura de coral muerto, roca arrecifal) y escombros (fragmentos de coral muerto, sustrato no consolidado). Para determinar qué variables ambientales contribuyen al entendimiento de la distribución de las especies de equinodermos se llevaron a cabo selecciones manuales (considerando no significativas aquellas cuyo valor de significancia sea mayor a 0.05). La significancia de los primeros ejes canónicos se corroboró mediante la prueba de Monte Carlo a 9999 permutaciones, y como criterio de corte se usó un alfa de 0.05. Dichos análisis se realizaron con el programa CANOCO v4.5 (Ter Braak \& Smilauer, 2003).

\section{RESULTADOS}

Se identificaron un total de 23 especies de equinodermos en el arrecife Enmedio, y están agrupados en cinco clases, 10 órdenes, 16 familias y 19 géneros. Las clases Ophiuroidea y Echinoidea fueron las 
Cuadro 1. Abundancia y densidad de las especies de equinodermos registrados en el arrecife Enmedio, Sistema Arrecifal Lobos-Tuxpan

Table 1. Abundance and density of echinoderm species recorded in Enmedio Reef, LobosTuxpan Reef System

\begin{tabular}{|c|c|c|c|c|c|c|c|c|}
\hline \multirow{2}{*}{ Especies } & \multicolumn{2}{|c|}{ Arrecife } & \multicolumn{2}{|c|}{ Sotavento } & \multicolumn{2}{|c|}{ Planicie } & \multicolumn{2}{|c|}{ Barlovento } \\
\hline & $\mathrm{A}$ & $\mathrm{D}$ & $\mathrm{A}$ & $\mathrm{D}$ & A & $\mathrm{D}$ & A & $\mathrm{D}$ \\
\hline \multicolumn{9}{|l|}{ Clase: Crinoidea } \\
\hline \multirow{2}{*}{\multicolumn{9}{|c|}{ Orden: Comatulida }} \\
\hline & & & & & & & & Familia: Comasteridae \\
\hline Davidaster rubiginosus (Pourtalès, 1869) & 3 & 0.01 & 3 & 0.02 & 0 & 0.00 & 0 & 0.00 \\
\hline \multicolumn{9}{|l|}{ Clase: Asteroidea } \\
\hline \multicolumn{9}{|l|}{ Orden: Valvatida } \\
\hline \multicolumn{9}{|l|}{ Familia: Ophidiasteridae } \\
\hline Copidaster lymani A. H. Clark, 1948 & 2 & 0.00 & 0 & 0.00 & 0 & 0.00 & 2 & 0.01 \\
\hline Linckia guildingi Gray, 1840 & 27 & 0.05 & 4 & 0.02 & 15 & 0.09 & 8 & 0.05 \\
\hline \multicolumn{9}{|l|}{ Orden: Forcipulata } \\
\hline \multicolumn{9}{|l|}{ Familia: Asteriidae } \\
\hline Coscinasterias tenuispina (Lamarck, 1816) & 5 & 0.01 & 2 & 0.01 & 0 & 0.00 & 3 & 0.02 \\
\hline \multicolumn{9}{|l|}{ Clase: Ophiuroidea } \\
\hline \multicolumn{9}{|l|}{ Orden: Ophiurida } \\
\hline \multicolumn{9}{|l|}{ Familia: Ophiactidae } \\
\hline Ophiactis savignyi (Müller \& Troschel, 1842) & 7 & 0.01 & 0 & 0.00 & 5 & 0.03 & 2 & 0.01 \\
\hline \multicolumn{9}{|l|}{ Familia: Ophiocomidae } \\
\hline Ophiocoma echinata (Lamarck, 1816) & 14 & 0.03 & 1 & 0.01 & 0 & 0.00 & 13 & 0.08 \\
\hline Ophiocomella ophiactoides (H. L. Clark, 1900) & 2 & 0.00 & 0 & 0.00 & 2 & 0.01 & 0 & 0.00 \\
\hline \multicolumn{9}{|l|}{ Familia: Ophiodermatidae } \\
\hline Ophioderma apressa (Say, 1825) & 4 & 0.01 & 4 & 0.02 & 0 & 0.00 & 0 & 0.00 \\
\hline Ophioderma cinerea Müller \& Troschel, 1842 & 15 & 0.03 & 1 & 0.01 & 0 & 0.00 & 14 & 0.08 \\
\hline \multicolumn{9}{|l|}{ Familia: Ophionereididae } \\
\hline Ophionereis reticulata (Say, 1825) & 1 & 0.00 & 0 & 0.00 & 1 & 0.01 & 0 & 0.00 \\
\hline \multicolumn{9}{|l|}{ Familia: Ophiuridae } \\
\hline Ophiolepis impressa Lütken, 1859 & 2 & 0.00 & 0 & 0.00 & 2 & 0.01 & 0 & 0.00 \\
\hline \multicolumn{9}{|l|}{ Familia: Ophiotrichidae } \\
\hline Ophiothrix (Ophiotrix) oerstedii Lütken, 1856 & 28 & 0.06 & 0 & 0.00 & 15 & 0.09 & 13 & 0.08 \\
\hline Ophiothrix (Acanthophiothrix) suensoni Lütken, & 10 & 0.02 & 3 & 0.02 & 2 & 0.01 & 5 & 0.03 \\
\hline
\end{tabular}

1856

Clase: Echinoidea

Orden: Cidaroida

Familia: Cidaridae

Eucidaris tribuloides (Lamarck, 1816)

Orden: Diadematoida

Familia: Diadematidae

Diadema antillarum Philippi, 1845

Orden: Temnopleuroida

Familia: Toxopneustidae

Lytechinus williamsi Chesher, 1968

Tripneustes ventricosus (Lamarck, 1816)

Orden: Echinoida

Familia: Echinoneidae

Echinometra lucunter lucunter (Linnaeus, 1758)

Echinometra viridis A. Agassiz, 1863

Clase: Holothuroidea

Orden: Dendrochirotida

Familia: Sclerodactylidae

Pseudothyone belli (Ludwig, 1887)

Orden: Aspidochirotida

Familia: Holothuriidae

Holothuria (Platyperona) parvula (Selenka, 1867) $\quad \begin{array}{lllllllll}1 & 0.00 & 0 & 0.00 & 1 & 0.01 & 0 & 0.00\end{array}$

Holothuria (Thymiosycia) arenicola Semper, $1868 \quad 1 \quad \begin{array}{llllllll}0.00 & 0 & 0.00 & 1 & 0.01 & 0 & 0.00\end{array}$

Familia: Stichopodidae

Isostichopus badionotus (Selenka, 1867)

\begin{tabular}{lccccccc}
21 & 0.04 & 17 & 0.10 & 0 & 0.00 & 4 & 0.02 \\
\hline
\end{tabular}

\begin{tabular}{llllllll}
5569 & 11.23 & 1477 & 8.94 & 2737 & 16.61 & 1355 & 8.21 \\
\hline
\end{tabular}




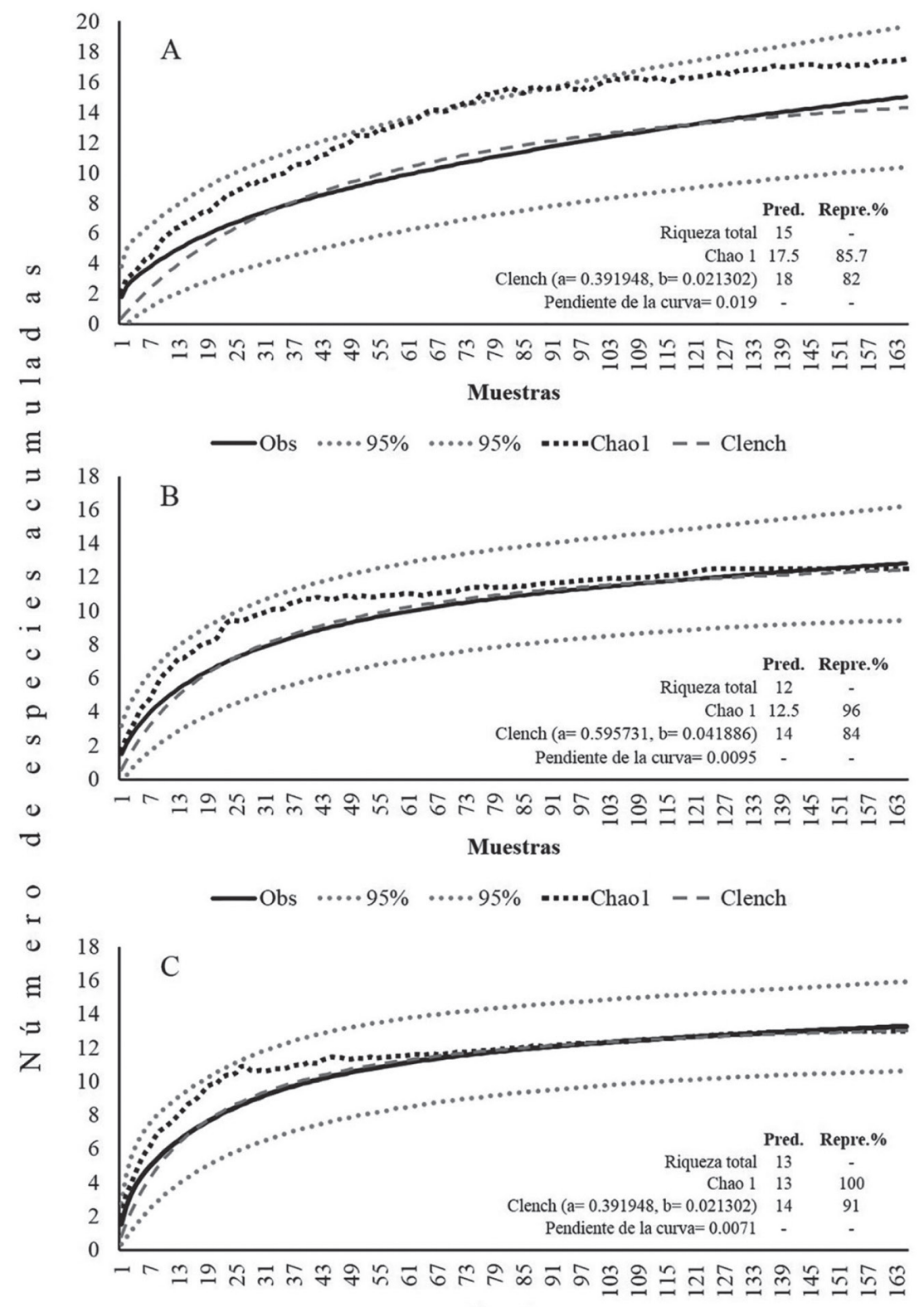

Muestras

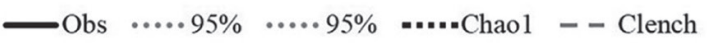

Fig. 2. Número de especies observadas (Obs) y estimadas (Chao 1 y Clench) para A) arrecife posterior, B) laguna arrecifal y C) arrecife frontal, correspondiente a la fauna de equinodermos del arrecife Enmedio, Sistema Arrecifal Lobos-Tuxpan. Las líneas punteadas negras indican el intervalo de confianza (superior e inferior) a 95\% del estimador Chao 1

Fig. 2. Number of species observed (Obs) and estimated (Chao 1 and Clench) for the A) back reef, B) reef lagoon and C) front reef, corresponding to the echinoderm fauna of the Enmedio Reef, Lobos-Tuxpan Reef System. Black lines indicate the 95\% confidence interval (upper and lower) of the Chao 1 estimator 
más representativas en riqueza con nueve y seis especies, respectivamente (Cuadro 1). Para la laguna arrecifal, se registraron 15 especies de equinodermos, sin embargo, la representatividad del muestreo fue de $86 \%$, dado que la predicción fue de 18 especies por parte del estimador Chao 1 (sesgo $=0.17$ y exactitud $=$ 0.01). El modelo de Clench estimó la misma riqueza de especies, por lo que la representatividad fue de $82 \%$, a pesar de ello la pendiente de la curva indica que el inventario faunístico es completo y fiable, además, las curvas acumulativas de especies muestran tendencia a la asíntota (Fig. 2A).

En el arrecife posterior, se registraron 12 especies de equinodermos, cifra que es cercana a la predicción de Chao 1 que fue de 12.5 especies estimadas $(\mathrm{sesgo}=0.041 \mathrm{y}$ exactitud $=0.003)$, por ende, la representatividad del muestreo fue de 96\%. Mientras, la predicción del modelo de Clench fue de 14 especies, esto significa que se registró el $86 \%$ de la riqueza, no obstante, la pendiente de la curva indica que también es confiable el esfuerzo de muestreo realizado, además, la curva acumulativa se aproximó a la asíntota (Fig. 2B). Con respecto al arrecife frontal, se encontraron 13 especies de equinodermos, esto coincide con la predicción de Chao 1, por lo que el esfuerzo de muestreo se considera bueno. El modelo de Clench indica que se obtuvo un $91 \%$ de representatividad, dado que la predicción fue de 14 especies, de manera que es confiable el inventario realizado, resultados que se observan en las curvas acumulativas de especies (Fig. 2C).
Por otra parte, la abundancia total fue de 5569 equinodermos, con una densidad de $11.23 \mathrm{ind} / \mathrm{m}^{2}$. La mayor abundancia se observó en la laguna arrecifal con 2737 individuos cuantificados, esto equivale a 16.61 ind $/ \mathrm{m}^{2}$. La clase Echinoidea fue el grupo con mayor número de individuos debido a que representó el 97.4\% de la fauna de equinodermos (Cuadro 1). Las especies más representativas fueron: Echinometra viridis con 3389 individuos y Echinometra lucunter lucunter con 1803 individuos, la densidad de estas especies fue de: 6.85 $\mathrm{ind} / \mathrm{m}^{2}$ y $3.64 \mathrm{ind} / \mathrm{m}^{2}$, respectivamente (Cuadro 1). También se registraron numerosos individuos de la clase Ophiuroidea representados por: Ophiothrix (Ophiothrix) oerstedii, Ophioderma cinerea y Ophiocoma echinata, asimismo, se contabilizaron cuantiosos asteroideos, esencialmente Linckia guildingi fue el más común de este grupo. Mientras Isostichopus badionotus como representante de la clase Holoturoidea fue la especie más abundante (Cuadro 1).

Para la de zona arrecifal, en el arrecife posterior (sotavento), la riqueza y la abundancia fueron de 12 especies y 1 477 individuos, respectivamente, mientras la densidad de equinodermos fue de $8.94 \mathrm{ind} / \mathrm{m}^{2}$, los equinoideos fueron $\mathrm{los}$ más representativos en abundancia y densidad, sobre todo $E$. viridis fue más abundante con 1248 individuos contabilizados, cifra que representa el $84 \%$ de la fauna de equinodermos para esta zona, le sigue Lytechinus williamsi con 150 individuos registrados, la densidad para ambas especies fue de $7.56 \mathrm{ind} / \mathrm{m}^{2}$ 


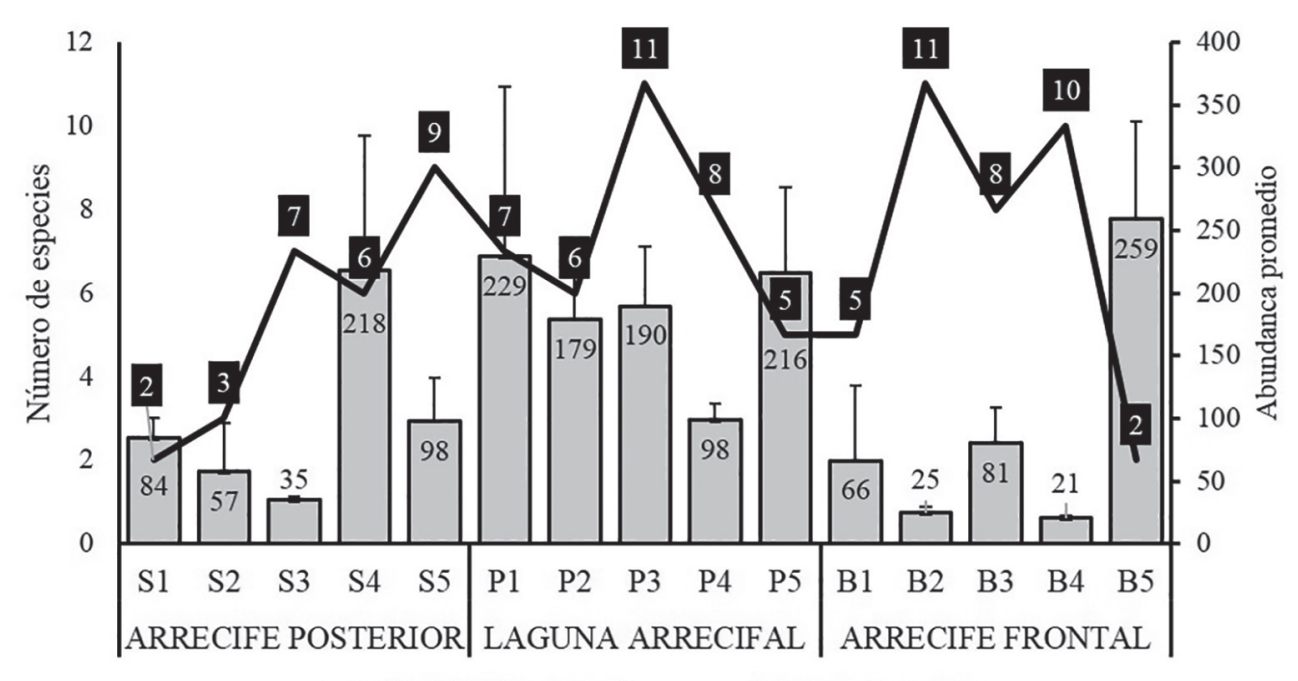

$\sqsubset$ Abundancia promedio $\longrightarrow$ Riqueza de especies

Fig. 3. Valores de riqueza de especies y abundancia promedio de los equinodermos del arrecife Enmedio, Sistema Arrecifal Lobos-Tuxpan. Los números arábigos indican los sitios de muestreo

Fig. 3. Values of species richness and average abundance of the Enmedio Reef echinoderms, Lobos-Tuxpan Reef System. Arabic numerals indicate sampling sites

y $0.91 \mathrm{ind} / \mathrm{m}^{2}$, respectivamente. Los sitios de menor riqueza y abundancia de equinodermos se observaron en la porción sur (S1) y en la porción suroeste de sotavento (S2), en estos lugares E. viridis fue el más numeroso; para la porción este (S3) y la porción norte (S5) también fue mínimo el número de equinodermos, sin embargo, se registraron mayor cantidad de especies, por el contrario, la abundancia promedio para la porción noroeste (S4) fue mayor a 200 individuos (Fig. 3), siendo E. viridis y L. williamsi los que más contribuyeron en la abundancia (Fig. 4). Las diferencias observadas entre los sitios son significativas para la abundancia ( $F=5.31$; $P=0.0148)$, pero no para la riqueza de especies $(F=4.60 ; P=0.230)$.

Para la laguna arrecifal, la riqueza fue de 15 especies y la abundancia de 2737 equinodermos, en esta zona se estimó una densidad de $16.61 \mathrm{ind} / \mathrm{m}^{2}$, las especies más representativas fueron: E. viridis y E. lucunter lucunter, quienes presentaron una abundancia de 1346 y 1326 individuos, respectivamente, esto equivale al $98 \%$ de la fauna registrada en esta zona arrecifal, mientras el resto de las especies registraron abundancias menores a 20 individuos; la densidad fue de $8.16 \mathrm{ind} / \mathrm{m}^{2}$ para $E$. viridis y de $8.04 \mathrm{ind} / \mathrm{m}^{2}$ para E. lucunter lucunter (Cuadro 1). De manera particular, la mínima riqueza fue de cinco especies registradas para el sitio P5 y la máxima fue de 11 especies para el sitio P3, sin embargo, estas diferencias no fueron significativas $(F=1.77 ; P=0.2119)$. La mayoría 

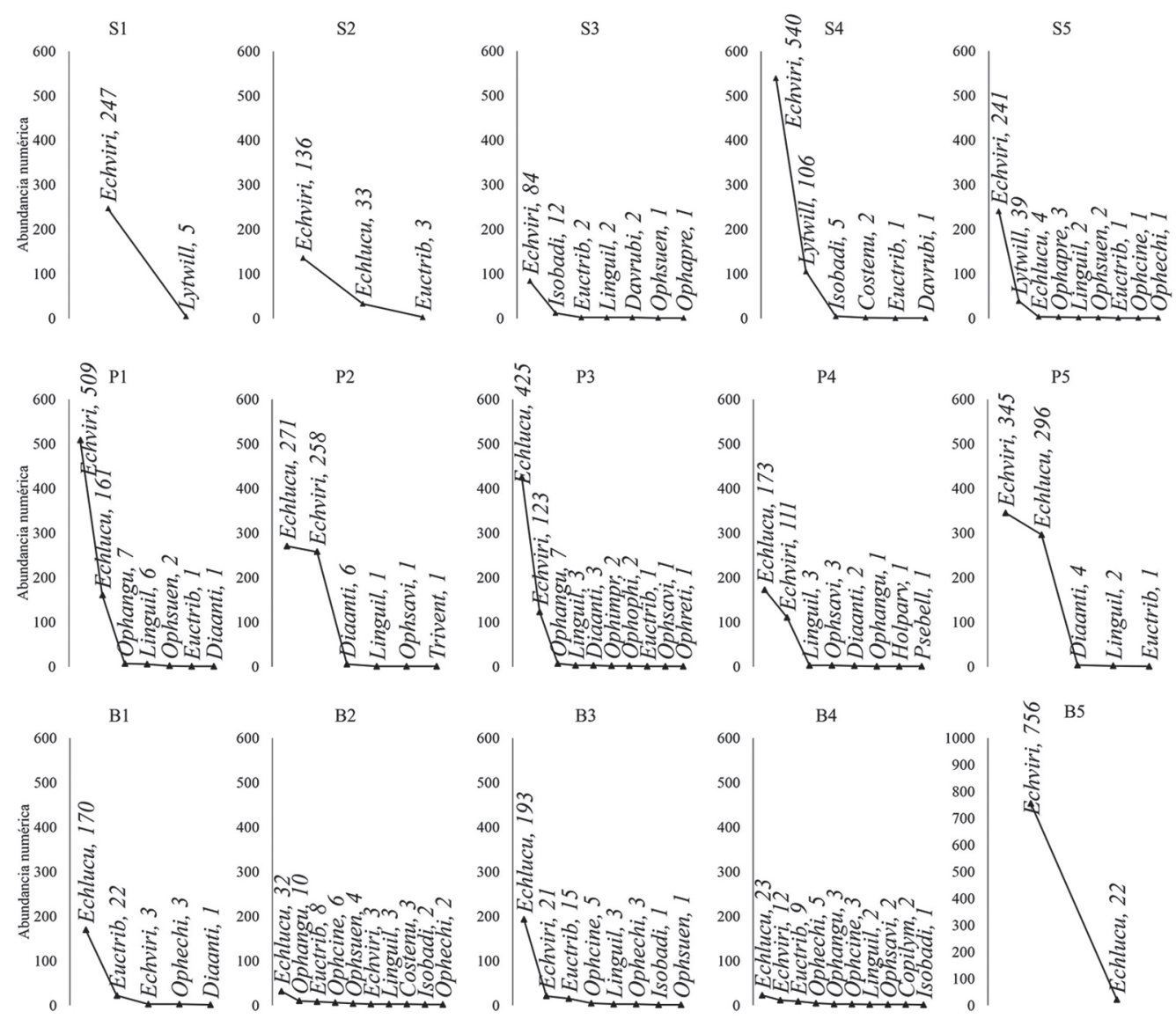

Fig. 4. Curvas de abundancia de las especies de equinodermos del arrecife Enmedio de los sitios y zonas de muestreo. Los nombres científicos se acortaron, las tres primeras letras corresponden al género y las cuatro últimas a la especie. Arrecife posterior (S), laguna arrecifal (P), arrecife frontal (B), los números arábigos indican los sitios de muestreo

Fig. 4. Abundance curves of the echinoderm species in Enmedio Reef at sites and sampling zones. Scientific names were shortened; the first three letters correspond to the genus and the last four to the species. Back reef (S), reef lagoon (P), front reef (B). Arabic numerals indicate sampling sites

de los sitios presentaron valores promedio de abundancia de $>150$ individuos, excepto en el sitio S4 donde se registraron en promedio 98 individuos (Fig. 3), estos contrastes tampoco fueron significativos $(F=1.33 ; P=0.324)$. A los extremos de planicie, en los sitios P1 y P5 se registró mayor abun- dancia de E. viridis, seguido de E. lucunter lucunter, mientras al interior de la laguna arrecifal en los sitios P2, P3 y $\mathrm{P} 4$, E. lucunter lucunter fue la más numerosa en individuos seguido de $E$. viridis (Fig. 4).

Para el arrecife frontal, se registró una riqueza de 13 especies y una 


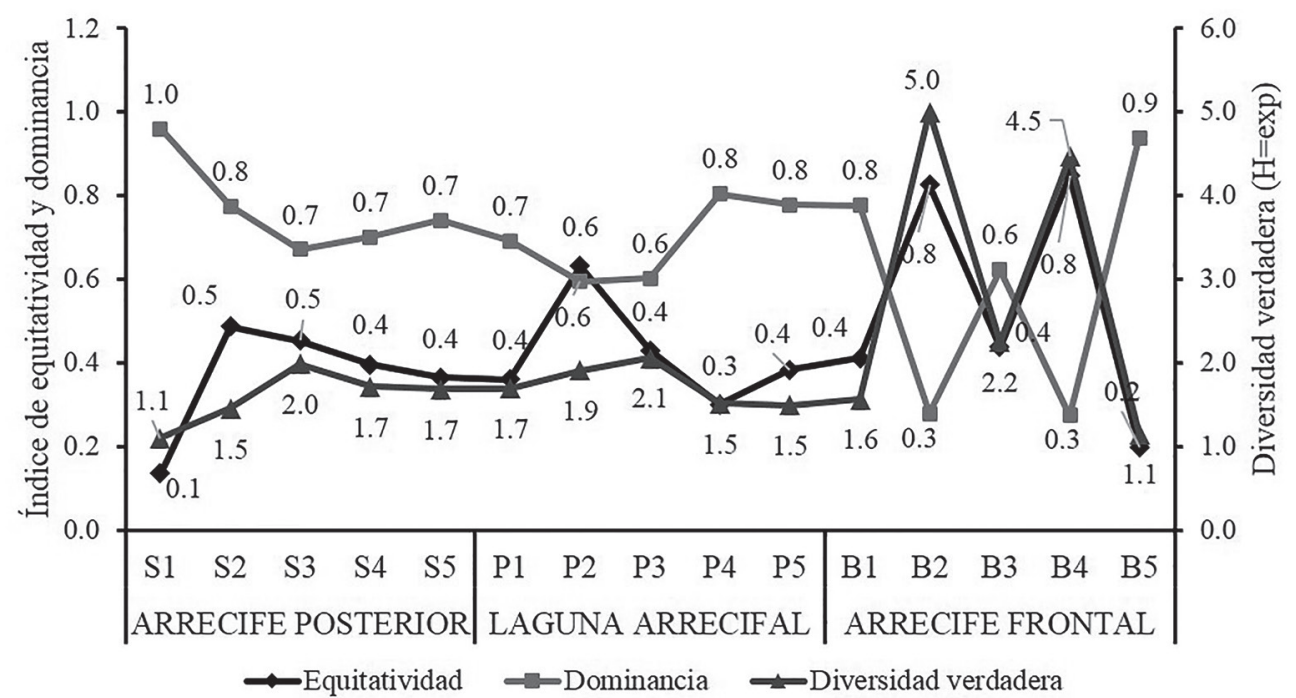

Fig. 5. Valores de equitatividad, dominancia y diversidad verdadera de los sitios de muestreo del arrecife posterior (S), laguna arrecifal (P) y arrecife frontal (B)

Fig. 5. Equity, dominance, and true diversity values of the back reef $(\mathrm{S})$, reef lagoon $(\mathrm{P})$ and front reef (B) sampling sites

abundancia de 1355 individuos, en esta zona los equinodermos presentaron una densidad de $8.21 \mathrm{ind} / \mathrm{m}^{2}$. Las especies de mayor abundancia fueron: E. viridis con 795 individuos, seguido de E. lucunter lucunter y de Eucidaris tribuloides con 440 y 54 organismos, respectivamente. Para esta zona arrecifal, la densidad de E. viridis fue de $4.82 \mathrm{ind} / \mathrm{m}^{2}$, para $E$. lucunter lucunter fue de $2.67 \mathrm{ind} / \mathrm{m}^{2}$ y para $E$. tribuloides fue de $0.03 \mathrm{ind} / \mathrm{m}^{2}$ (Cuadro 1). En los primeros sitios: B1, B2, B3 y B4, se registraron abundancias promedio $<100$ individuos, mientras la mínima riqueza se registró en el sitio B2 con cinco especies y como máximo se encontraron 10 especies en el sitio B4 (Fig. 3), en estos lugares de muestreo $E$. lucunter lucunter fue la más abundante de las especies registradas, por el contrario el sitio con menor riqueza, pero con mayor abundancia promedio, fue el sitio B5 (Fig. 3), las especies cuantificadas en este lugar fueron: E. viridis y E. lucunter lucunter con 756 y 22 individuos, respectivamente (Fig. 4). Las diferencias observadas entre los sitios son significativas para la riqueza de especies $(F=4.42 ; P=0.257)$ y la abundancia de equinodermos $(F=$ $13.88 ; P=0.0004)$.

En relación con los índices ecológicos, los sitios del arrecife frontal presentaron valores altos de dominancia, sobre todo en los sitios $\mathrm{S} 1$ y S2 con valores de $D=1$ y $D=0.8$, respectivamente, en los demás sitios fue de $D=0.7$, esto explica el bajo número de especies efectivas $\left({ }^{1} D\right)$ en todos los sitios de sotavento, dado que se registraron entre 0.1 (S1) 
A

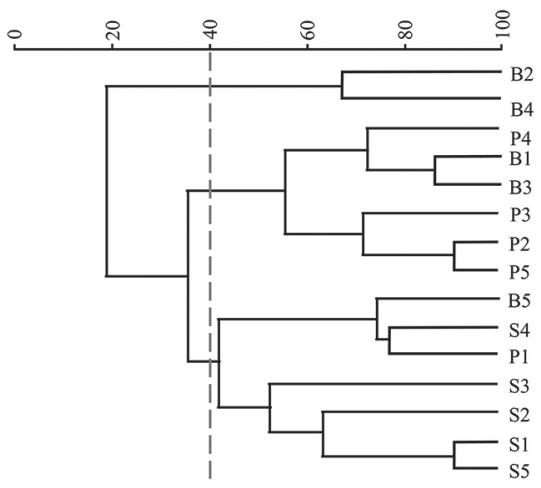

B

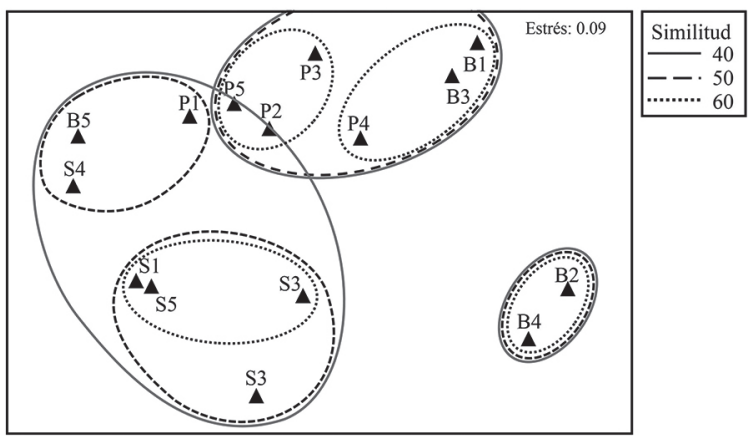

Fig. 6. A) Análisis de clasificación y B) ordenamiento multidimensional no métrico basado en la distancia de Bray-Curtis aplicado a los sitios del arrecife posterior (S), laguna arrecifal (P) y arrecife frontal (B)

Fig. 6. A) Classification analysis and B) non-metric multidimensional ordering based on Bray-Curtis distance applied to the back reef $(\mathrm{S})$, reef lagoon $(\mathrm{P})$, and frontal reef $(\mathrm{B})$ sites

Cuadro 2. Especies de equinodermos que contribuyeron en la similitud de los sitios de muestreo, Arrecife Enmedio, Sistema Arrecifal Lobos-Tuxpan

Table 2. Species of echinoderms that contributed to the similarity of the sampling sites, Enmedio Reef, Lobos-Tuxpan Reef System

\begin{tabular}{llll}
\hline Especies & Similitud promedio & \% contribución & \% acumulado \\
\hline E. viridis & $26.21 \pm 1.48$ & 54.27 & 54.27 \\
E. lucunter lucunter & $14.29 \pm 0.97$ & 29.59 & 83.87 \\
\hline
\end{tabular}

y 2 especies verdaderas (S3) para esta zona arrecifal (Fig. 5), estadísticamente no hay diferencias significativas en los valores de equitatividad $(F=1.22 ; P=$ $0.3605)$ y dominancia $(F=2.05 ; P=$ $0.1636)$. En la laguna arrecifal, también prevaleció la dominancia, los sitios P4 y P5 presentaron mayor valor en este atributo con $D=0.8$, el resto de los sitios registraron valores de entre $D=0.6 \mathrm{y}$ $D=0.7$, en consecuencia, la diversidad verdadera fue mínima en estos lugares de la planicie, registrándose como máximo
2.1 especies efectivas $\left({ }^{1} D\right)$ en el sitio P3 (Fig. 5), de igual manera el análisis de varianza determinó que no hay diferencias estadísticas en los valores de equitatividad $(F=1.39 ; P=0.3053)$ y dominancia $(F=1.38 ; P=0.307)$. En el arrecife frontal, la dominancia se registró únicamente en los sitios $\mathrm{B} 1(D=0.8)$, B3 $(D=0.6)$ y B5 $(D=0.9)$, asimismo, en estos lugares la diversidad verdadera $\left({ }^{1} D\right)$ fue mínima, por el contrario en los sitios B2 y B4 se registraron valores altos en equitatividad $(\mathrm{J}=0.8)$, por 
consiguiente, estos lugares fueron los más diversos en especies efectivas $\left({ }^{1} D\right)$ en comparación con el resto de los sitios del arrecife frontal, laguna arrecifal y arrecife posterior (Fig. 5). Para esta zona, las diferencias observadas entre los sitios son significativas tanto en la equitatividad $(F=7.79 ; P=0.0041)$ como en la dominancia $(F=7.66 ; P=0.0043)$.

En relación con el análisis de similitud de Bray-Curtis, haciendo un corte al $40 \%$ se diferencian tres grupos. El primero lo componen los sitios de barlovento: B2 y B4, el segundo conjunto lo representan dos sitios de barlovento: B1 y B3 y cuatro sitios de planicie: P2, P3, P4, P5, la tercera agrupación la componen los cinco sitios de sotavento, un sitio de planicie: P1 y un sitio de barlovento: B5 (Fig. 6A). El método nMDS muestra las proximidades de los sitios estudiados, separándose con claridad los sitios B2 y B4 de barlovento (Fig. 6B), sin embargo, el análisis SIMPROF no detectó grupos significativamente diferentes, el análisis SIMPER determinó que dos especies contribuyen a la similitud de los grupos formados (Cuadro 2).

El ACC muestra que al menos diez especies de equinodermos presentaron relaciones positivas con cuatro sustratos bentónicos. Las especies E. viridis, Pseudothyone belli y Holothuria (Platyperona) parvula manifestaron afinidad positiva con la cobertura de corales y negativa con la cobertura de algas filamentosas y escombros que caracterizan la laguna arrecifal; por otro lado, L. guildingi presentó preferencia por la cobertura de macroalgas frondosas, en cambio E. lucunter lucunter y Tripneustes ventricosus exhibieron mayor correspondencia con la cobertura de escombros y algas filamentosas, otras especies mostraron una relación débil con estas variables ambientales: Ophiactis savignyi, Ophionereis reticulata, Holothuria (Thymiosycia) arenicola y Diadema antillarum. El resto de las especies de equinodermos no manifestaron ninguna relación con los sustratos bentónicos analizados (Fig. 7).

\section{DISCUSIÓN}

En la laguna del arrecife Enmedio parece ser habitual la notable abundancia de equinodermos, dado que resultados similares se han reportado para el arrecife Lobos (De la Cruz-Francisco, 2013) y en seis arrecifes del SAV (Pérez-España et al. 2015). No obstante, E. viridis superó en abundancia a $E$. lucunter lucunter, resultado que es contrario a lo que se reporta para la planicie del arrecife Lobos (De la Cruz-Francisco, 2013) y en Isla Verde, perteneciente al SAV (CelayaHernández et al. 2008), ya que en estos ecosistemas citan a $E$. lucunter lucunter como la más abundante. Los resultados obtenidos sugieren que ambas especies cohabitan en la planicie arrecifal, esto puede atribuirse a que muestran las mismas preferencias por sustratos rocosos y coralinos rocosos (CelayaHernández et al. 2008), componentes bentónicos que son comunes en la laguna del arrecife Enmedio (GonzálezGonzález et al. 2016).

Fundamentalmente, E. viridis fue 


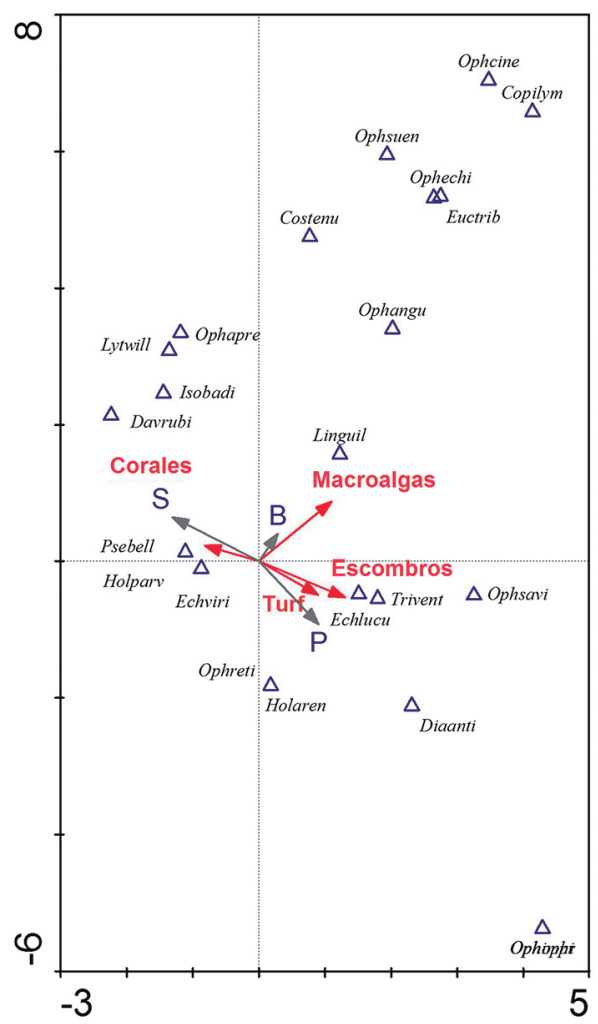

Fig. 7. Análisis de correspondencia canónica aplicado a las especies de equinodermos del arrecife Enmedio, Sistema Arrecifal Lobos-Tuxpan. Las variables ambientales corresponden a la cobertura bentónica del mismo arrecife

Fig. 7. Canonical correlation analysis applied to the echinoderm species of Enmedio Reef, Lobos-Tuxpan Reef System. The environmental variables correspond to the reef's benthic cover

más abundante en sitios (P1 y P5) que confluyen a las pendientes profundas con mayor cobertura coralina $y$ pavimento coralino (GonzálezGonzález et al. 2016), de hecho se ha mencionado que la abundancia de E. viridis se correlaciona con la cobertura coralina (Bologna et al.
2012). Mientras E. lucunter lucunter fue más abundante al interior de la laguna, específicamente en sitios ( $\mathrm{P} 2$, P3 y P4) más superficiales expuestos al embate de las olas donde prevalecen abundantes corales (GonzálezGonzález et al. 2016) que presentan plasticidad morfológica (Millepora alcicornis, Pseudodiploria clivosa, Porites furcata, Orbicella annularis) para tolerar el estrés ambiental como el oleaje y la intensidad de la luz (Kaandorp, 1999; LaJeunesse, 2002; Darling et al. 2012) y, precisamente, E. lucunter lucunter es considerada también como una especie que soporta el estrés ambiental (GaitánEspitia, 2008) que impera en la planicie arrecifal (Jordán-Dahlgren, 1993). Esta capacidad le posibilitaría prosperar y ser más abundante sobre todo en ambientes someros, debido a que tiende a decrecer en número a profundidades $>10 \mathrm{~m}$ (Gaitán-Espitia, 2008; Cordeiro et al. 2014).

Con base en los índices ecológicos, en la laguna arrecifal los equinodermos presentaron tendencia a la dominancia y no a la equitatividad, esto puede atribuirse a la notable abundancia que presentaron E. viridis y E. lucunter lucunter, y explicar la baja diversidad verdadera en todos los sitios de muestreo. El sitio P4 presentó mayor valor de dominancia, debido a que $E$. lucunter lucunter fue más abundante que E. viridis. Además, en este lugar la abundancia de equinodermos fue mínima comparada con los restantes sitios de muestreo de la planicie, estos resultados probablemente se relacionan 
con las características del sustrato, dado que abundan escombros y dos especies incrustantes: Erytropodium caribaeorum y Palythoa caribaeorum (González-González et al. 2016). Estas especies son competidoras por el espacio y de rápido crecimiento (Sebens \& Miles, 1988; Silva et al. 2015) y podrían considerarse como desfavorables para los erizos, ya que no proveen refugios adecuados como grietas y oquedades, atributos que sí proporcionan los corales, pero que manifestaron baja cobertura en dicho sitio. En cambio la mayor abundancia de equinodermos fue justamente en los demás sitios de la planicie que presentan importantes valores de cobertura coralina (GonzálezGonzález et al. 2016).

En las zonas profundas del arrecife, la abundancia de equinodermos fue menor comparada con la planicie, resultado que coincide con lo reportado para el arrecife Lobos (De la CruzFrancisco, 2013) y en los arrecifes del SAV (Pérez-España et al. 2015). Particularmente, en el arrecife posterior, si bien se encontraron 12 especies de equinodermos, la comunidad está representada por la considerable abundancia de $E$. viridis en todos los sitios de muestreo, es probable que la abundancia de esta especie se correlacione en forma positiva con la cobertura coralina (Lessios, 1998; Bologna et al. 2012), y precisamente los corales manifiestan su máximo desarrollo en esta zona (González-González et al. 2016). La otra especie de mayor abundancia fue $L$. williamsi, quien puede considerarse típica de sotavento, dado que no fue observada en la planicie ni en barlovento. Esta especie fue abundante en los sitios S4 y S5, lugares donde los corales masivos son abundantes, representados por $O$. annularis (González-González et al. 2016). En el arrecife Lobos también se ha reportado su presencia, asimismo, en el arrecife posterior cohabitando con $E$. viridis (De la Cruz-Francisco, 2013; González-Gándara et al. 2015).

En los primeros sitios de sotavento (S1 y S2) la riqueza de especies y la abundancia de equinodermos fueron menores, esto puede atribuirse a las características del sustrato, dado que en estos lugares la pendiente es corta y abrupta, y es considerable la presencia de arena y pavimento coralino (González-González et al. 2016), los corales (C. natans, O. annularis, $M$. cavernosa) que se desarrollan en estos sitios probablemente proveen refugios que en especial son ocupados por E. viridis. Al ser esta la especie más abundante, condiciona una comunidad dominante en estos lugares. Cabe mencionar que en el sitio S3 la dominancia decreció, de igual manera la abundancia de equinodermos fue la más baja a pesar de que se reporta mayor cobertura coralina (GonzálezGonzález et al. 2016), esto sugiere que la disponibilidad de refugios es menor para los equinoideos, y puede ser atribuida a la abundancia de $C$. natans, las colonias de esta especie de coral son en forma de domo, por lo que su estructura coralina no proveería refugios potenciales. En los sitios 
S4 y S5, la dominancia prevalece en la comunidad de equinodermos por la notable abundancia de E. viridis, en estos lugares la abundancia y la riqueza de especies son mayores. Todo pareciera indicar que se debería a la considerable cobertura de $O$. annularis (González-González et al. 2016), dado que los corales de esta especie son grandes, masivos y en especial presentan lóbulos separados, espacios que oportunamente son ocupados como hábitats por los equinodermos. Esta peculiaridad que presenta $O$. annularis en proveer hábitats para una vasta fauna ha sido destacada en varios trabajos (Mumby et al. 2008; Harborne et al. 2011; 2012; De la Cruz-Francisco et al. 2016a).

Contrariamente, en el arrecife frontal se registró menor abundancia de equinodermos y podría atribuirse a la baja cobertura coralina. Esta zona se caracteriza por la prevalencia de pavimento coralino, algas coralinas costrosas, octocorales y algas filamentosas (González-González et al. 2016). Estos componentes bentónicos también podrían considerarse como desfavorables para la abundancia de equinodermos al no proveer oquedades o grietas, espacios que son importantes principalmente para los erizos, con el fin de protegerse de la fuerza del oleaje que es común en esta zona arrecifal (Jordán-Dahlgren, 1993). La baja abundancia de equinoideos posibilita una mayor riqueza de especies $y$, por consiguiente, una mayor equitatividad como la registrada en los sitios B2 y B4, no obstante, a los extremos del arrecife frontal, la comunidad sí tiende a ser dominante por la notable abundancia de E. lucunter lucunter en el sitio B1 y de E. viridis en el sitio B5. Aunado a lo anterior, los corales parecen desempeñar un papel clave en la abundancia de equinodermos, tal como ocurre en la laguna arrecifal $y$ en el arrecife posterior, zonas donde la cobertura coralina es mayor a $>15 \%$ (González-González et al. 2016) y la abundancia de equinodermos es igualmente representativa de acuerdo con los datos obtenidos.

Cabe mencionar que también se contabilizaron tres individuos de Davidaster rubiginosus esencialmente en la ladera de sotavento, esta especie de crinoideo es la única que se cita para los arrecifes coralinos del Estado (Solís-Marín et al. 2007; De la CruzFrancisco, 2013; González-Gándara et al. 2015), las demás especies que se reportan para el Golfo de México se distribuyen en aguas más profundas >50 m (Durán-González et al. 2005; Pawson et al. 2009). Davidaster rubiginosus es de hábitos crípticos y se resguarda entre las grietas de corales y roca coralina, presenta un rango de distribución batimétrica de 3-50 m de profundidad (Pawson et al. 2009), razón por la cual no fue observada en la planicie arrecifal.

También se registraron numerosos asteroideos preferentemente en la planicie, representados por poblaciones de $L$. guildingi, esta especie se caracteriza por tener una gran capacidad de regeneración (Cortés-Rivera et al. 2016), suele encontrarse a profundi- 
dades $<10 \mathrm{~m}$, habitando en sustratos duros y en parches de arena (Hendler et al. 1995; Solís-Marín et al. 2007; Gaitán-Espitia, 2008), justamente estos hábitats bentónicos son característicos en el arrecife Enmedio (De la Cruz-Francisco et al. 2016; González-González et al. 2016). También se observaron individuos en las zonas profundas $>10 \mathrm{~m}$, lo cual se atribuye a que puede habitar hasta profundidades $>200 \mathrm{~m}$ (Pawson et al. 2009).

De igual manera se contabilizaron organismos de la clase Ophiuroidea, si bien se identificaron nueve especies, solo Ophiocoma echinata y Ophioderma cinerea fueron comunes en barlovento, mientras en la planicie fue abundante Ophiothrix oerstedii. Estas tres especies se encontraron debajo de rocas $\mathrm{y}$ en escombros, probablemente porque son de hábitos crípticos (Hendler et al. 1995), no obstante, O. oerstedii, así como el resto de Ophiotrix spp. registrados en el estudio son capaces de ocupar otros hábitats como macroalgas (Boffi, 1972) y esponjas (Hendler, 1984; Carrera-Parra \& Vargas-Hernández, 1996; Henkel \& Pawlik, 2005; 2011). Especies cuantificadas en el estudio $(<10$ individuos) como Ophiothrix angulata, Ophiactis savignyi, Ophiocomella ophiactoides, O. echinata y Ophionereis reticulata son citadas como abundantes en Venezuela (Gómez-Maduro \& Hernández-Ávila, 2015) y Colombia (Gaitán-Espitia, 2008).

De acuerdo con los análisis multivariados, la composición y la abundancia de equinodermos manifestaron baja similitud entre los sitios de muestreo, pero no fueron significativos, las especies que contribuyeron en la similitud de los sitios fueron precisamente las más abundantes: E. viridis y E. lucunter lucunter. Estas especies están bien representadas en las tres zonas arrecifales, por ello, los equinodermos no manifestaron una clara zonación en el arrecife. Estos resultados obtenidos no coinciden con el patrón de distribución general que se describe para los arrecifes del polígono Lobos (González-Gándara et al. 2015). Inclusive, en esta área de estudio, se ha demostrado que especies sésiles como esponjas y corales sí exhiben una marcada zonación, siendo el arrecife posterior la zona que concentra mayor riqueza de especies y cobertura coralina (De la Cruz-Francisco et al. 2016a, b; González-González et al. 2016). Estos contrastes sugieren que probablemente los equinodermos responden a otros factores como las características del sustrato y las preferencias alimenticias (Hendler et al. 1995; Celaya-Hernández et al. 2008; Bologna et al. 2012).

El análisis de correspondencia canónica reveló que solo diez especies de equinodermos presentaron asociaciones propias en función de la disponibilidad de refugio/alimento. $E$. viridis mostró mayor asociación con la cobertura coralina, resultado que coincide con varios trabajos previos (Lessios, 1998; Celaya-Hernández et al. 2008; Cordeiro et al. 2014). En apariencia se refugia preferentemente en Agaricia, Pseudodiploria, 
Montastraea (Lessios, 1998), estos corales son comunes en el arrecife Enmedio (González-González et al. 2016). También $P$. belli y $H$. parvula mostraron afinidad por los corales, estos holotúridos son de talla pequeña, por lo que suelen habitar en los intersticios de rocas y de corales (Hendler et al. 1995). Mientras L. guildingi presentó mayor asociación con la cobertura de macroalgas, que probablemente corresponda con el hábito alimenticio, dado que la literatura menciona que esta especie consume organismos adheridos al sustrato como protozoarios, bacterias y algas (Hendler et al. 1995; Stropes, 2003), además, se ha mencionado que puede presentar hábitos detritívoros y carnívoros (Stropes, 2003).

Por otra parte, E. lucunter lucunter mostró principalmente asociación con los escombros y las algas filamentosas, estos componentes bentónicos son abundantes en los lugares más superficiales de la laguna arrecifal (González-González et al. 2016), donde el estrés ambiental limita la presencia de organismos arrecifales. Precisamente, E. lucunter lucunter es capaz de soportar el embate de las olas, capacidad que le permite prosperar y ser abundante (McClanahan \& Muthiga, 2013). Otro significado de esta asociación parece estar ligado a cuestiones tróficas, dado que la alimentación primaria de E. lucunter lucunter son las algas (Hendler et al. 1995). Así también, $T$. ventricosus mostró asociación con los escombros, esto puede atribuirse a que los organismos encontrados debajo de restos coralinos eran de talla pequeña, ya que habitan en los pastos marinos (Lawrence \& Agatsuma, 2013; González-Gándara et al. 2015). Mientras D. antillarum, O. savignyi, Holothuria arenicola y O. reticulata son especies que se distribuyeron en la planicie, pero mostraron una relación débil con las algas filamentosas y escombros, probablemente por la baja abundancia que manifestaron en esta zona arrecifal. A excepción de $D$. antillarum, las demás especies citadas antes en general son de hábitos crípticos y suelen habitar en las frondas de algas y en intersticios de restos de coral muerto, lo que dificulta contabilizarlas en los censos visuales.

En definitiva, la clase Echinoidea caracteriza la fauna de equinodermos del arrecife Enmedio representados fundamentalmente por E. lucunter lucunter y E. viridis. En la laguna arrecifal fue notable la abundancia y densidad de equinodermos, sin embargo, en los tres ambientes arrecifales la diversidad verdadera fue mínima y con valores altos de dominancia de Simpson. La baja similitud entre los sitios de muestreo no fue significativa y no manifestó un patrón de zonación característico y/o similar a lo que manifiestan organismos sésiles como esponjas y corales, lo cual puede estar ligado a que son organismos móviles y pueden desplazarse en el arrecife para buscar refugio y alimento, tal como se determinó en el estudio donde diez especies de equinodermos manifestaron afinidades propias de acuerdo con la cobertura de 
macroalgas, algas filamentosas, corales y escombros, componentes bentónicos que son importantes al proveer refugio y alimentación. Con esta información se amplía el conocimiento ecológico de los equinodermos para los arrecifes de Veracruz, esta puede servir de base para la toma de decisiones en materia de manejo y protección. Asimismo, es fundamental continuar investigando en los demás arrecifes coralinos que conforman el SALT, dado que aún se desconocen la composición y la abundancia de equinodermos en los arrecifes sumergidos Pantepec y Oro Verde.

\section{AGRADECIMIENTOS}

Agradecemos a Irving Abad Aguilar, por su apoyo en el trabajo de campo. A Rosa Estela Orduña Medrano, por su apoyo en la revisión y corrección del Abstract. Al personal que labora en el Club Náutico Tampamachoco y Club Náutico Aqua Sport, por el apoyo que siempre mostraron al recibirnos y atendernos en todo momento. A los revisores anónimos por sus comentarios y sugerencias.

\section{BIBLIOGRAFÍA}

Benavides-Serrato, M., Borrero-Pérez, G. H. \& Díaz-Sánchez, C. M. (2011). Equinodermos del Caribe Colombiano I: Crinoidea, Asteroidea y Ophiuroidea. Santa Marta. Colombia. Serie de publicaciones especiales de Invemar 22.

Boffi, E. (1972). Ecological aspects of ophiuroids from the phytal of S. W.
Atlantic Ocean warm waters. Mar. Biol., 15, 316-328.

Bologna, P. A. X., Webb-Wilson, L., Connelly, P. \& Saunders, J. E. (2012). A new baseline for Diadema antillarum, Echinometra viridis, E. lucunter, and Eucidaris tribuloides populations within the Cayos Cochinos, MPA, Honduras. Gulf and Caribbean Research, 24(1), 1-5.

Borrero-Pérez, G. H., Benavides-Serrato, M. \& Díaz-Sánchez, C. M. (2012). Equinodermos del Caribe Colombiano II: Echinoidea y Holothuroidea. Santa Marta. Colombia. Serie de publicaciones especiales de Invemar 30.

Carrera-Parra, L. F. \& Vargas-Hernández, J. M. (1996). Comunidad críptica de esponjas del arrecife de Isla de Enmedio, Veracruz, México. Rev. Biol. Trop., 44(3), 311-321.

Caso, M. E. (1994). Estudio morfológico, taxonómico, ecológico y distribución geográfica de los asteroideos colectados durante las campañas oceanográficas Cortés 1 . 2. 3. An. Inst. Cienc. Mar Limnol. UNAM, 12, 1-111.

Celaya-Hernández, E. V., Solís-Marín, F. A., Laguarda-Figueras, A., DuránGonzález, A. de la L. \& Ruiz, R. T. (2008). Asociación a sustratos de los erizos regulares (Echiodermata: Echinoidea) en la laguna arrecifal de Isla Verde, Veracruz, México. Rev. Biol. Trop., 56(Supl. 3), 281-295.

Chávez, E. A., Tunnell, J. W. Jr. \& Withers, K. (2010). Zonación y Ecología de los Arrecifes: Plataforma 
Veracruzana y Banco Campeche. En J. W. Tunnell Jr., E. A. Chávez \& K. Withers (Eds.), Arrecifes Coralinos del Sur del Golfo de México (pp. 60-100). México: Instituto Politécnico Nacional.

Clarke, K. R. \& Gorley, R. N. (2015). Getting started with PRIMER V7. PRIMER-E. United Kingdom. West Hoe Plymouth PL1 3 DH. PRIMER-E Ltd.

Colwell, R. K. (2013). EstimateS: Statistical estimation of species richness and shared species from samples. Version 9. Recuperado el 9 de agosto, 2017, disponible en http://viceroy.colorado.edu/estimates/index.html

Cordeiro, C. A. M. M., Harborne, A. R. \& Ferreira, C. E. L. (2014). Patterns of distribution and composition of sea urchin assemblages on Brazilian subtropical rocky reefs. Mar. Biol., 161(10), 2221-2232.

Cortés-Rivera, Y., Hernández, R. I., San Martín del Ángel, P., Zarza, M. E. \& Cuervo-González, R. (2016). Potencial regenerativo de la estrella de mar Linckia guildingi. Hidrobiológica, 26(1), 103-108.

Darling, E. S., Álvarez-Filip, L., Oliver, T. A., McClanahan, T. R. \& Coté, I. M. (2012). Evaluating life history strategies of reefs corals from species traits. Ecol. Lett., 15, 1378-1386.

De la Cruz-Francisco, V. (2013). Estructura de las comunidades macrobentónicas y nectónicas asociadas a los sustratos rocosos coralinos del arrecife Lobos, Veracruz, México. Tesis de maestría no publicada,
Facultad de Ciencias Biológicas y Agropecuarias, Veracruz, México.

De la Cruz-Francisco, V., GonzálezGonzález, M. \& Flores-Galicia, L. (2016a). Distribución de los hábitats bentónicos de la laguna del Arrecife Enmedio, Sistema Arrecifal Lobos-Tuxpan, México. Rev. Invest. Mar., 36(1), 63-78.

De la Cruz-Francisco, V., GonzálezGonzález, M. \& Morales-Quijano, I. (2016b). Faunística y distribución de Demospongiae: Porífera del arrecife Enmedio, Sistema Arrecifal Lobos-Tuxpan, México. CICIMAR Oceánides, 31(1), 7-16.

De la Cruz-Francisco, V., GonzálezGonzález, M. \& Morales-Quijano, I. (2016c). Inventario taxonómico de Hydrozoa (Orden: Anthoathecata) y Anthozoa (Subclases: Hexacorallia y Octocorallia) del Arrecife Enmedio, Sistema Arrecifal LobosTuxpan. CICIMAR Oceánides, 31(1), 23-34.

Durán-González, A., Laguarda-Figueras, A., Solís-Marín, F. A., Sánchez, B. B., Ahearn, C. G. \& Torres-Vega, J. (2005). Equinodermos (Echinodermata) de las aguas mexicanas del Golfo de México. Rev. Biol. Trop., 53(Suppl. 3), 53-68.

Gaitán-Espitia, J. D. (2008). Estructura de la comunidad del Phylum Echinodermata en aguas someras de la bahía de Taganga, Caribe Colombiano. Rev. U. D. C. A. Act. Div. Cient., 11(1), 85-93.

Gómez-Maduro, M. C. \& HernándezÁvila, I. (2015). Comunidades de 
equinodermos en la región de Tunantal, Golfo de Cariaco, Venezuela. Rev. Biol. Trop., 63(Suppl. 2), 195-207.

González-Azcárraga, A. (2009). Estructura de las Asociaciones y Diversidad morfológica de erizos de mar (Echinoidea) en los Parques Nacionales Sistema Arrecifal Veracruzano y Arrecifes de Cozumel, México. Tesis de maestría no publicada, Universidad Nacional Autónoma de México, Sinaloa, México. González-Gándara, C., Solís-Marín, F. A., De la Cruz-Francisco, V., Granados-Barba, A., Salas-Pérez, J. D. J., Argüelles-Jiménez, J. \& Escárcega-Quiroga, P. A. (2015). Riqueza y distribución de equinodermos en los arrecifes del norte y sur de Veracruz, México. Rev. Biol. Trop., 63(2), 183-193.

González-González, M., De la CruzFrancisco, V., Morales-Quijano, I. \& Orduña-Medrano, R. E. (2016). Diversidad y cobertura de la comunidad bentónica del arrecife Enmedio, Sistema Arrecifal Lobos-Tuxpan, México. Rev. Mar. Cost., 8(2), 47-65.

Harborne, A. R., Mumby, P. J. \& Ferrari, R. (2012). The effectiveness of different meso-scale rugosity metrics for predicting intra-habitat variation in coral-reef fish assemblages. Env. Biol. Fish., 94(2), 431-442.

Harborne, A. R., Mumby, P. J., Kennedy, E. V. \& Ferrari, R. (2011). Biotic and multi-scale abiotic controls of habitat quality: their effect on coral-reef fishes. Mar. Ecol. Prog. Ser., 437, 201-214.

Hendler, G. (1984). The association of Ophiotrix lineata and Callyspongia vaginalis: a brittlestar-sponge cleaning symbiosis? Mar. Ecol., 5(1), 9-27.

Hendler, G., Miller, J. E., Pawson, D. L. \& Kier, P. M. (1995). Sea Stars, Sea Urchins and Allies: Echinoderms of Florida and the Caribbean. Washington: Smithsonian Institution Press.

Henkel, T. P. \& Pawlik, J. R. (2005). Habitat use by sponge-dwelling brittlestars. Marine Ecology, 146, 301-313.

Henkel, T. P. \& Pawlik, J. R. (2011). Host specialization of an obligate sponge-dwelling brittlestar. Aquat. Biol., 12, 37-46.

Hernández-Herrejón, L. A., Solís-Marín, F. A. \& Laguarda-Figueras, A. (2008). Ofiuroideos (Echinodermata: Ophiuroidea) de las aguas mexicanas del golfo de México. Rev. Biol. Trop., 56(3), 83-167.

Hill, J. \& Wilkinson, C. L. I. V. E. (2004). Methods for ecological monitoring of coral reefs. Townsville: Australian Institute of Marine Science.

Humann, P. \& Deloach. N. (2002). Reef Creature identification Florida Caribbean Bahamas (2nd. ed.). California: New World Publications.

Jiménez-Valverde, A. \& Hortal, J. (2003). Las curvas de acumulación de especies y la necesidad de evaluar la calidad de los inventarios biológicos. Rev. Iber. Aracnol., 8, 151-161. 
Jordán-Dahlgren, E. (1993). El ecosistema arrecifal coralino del Atlántico Mexicano. Rev. Soc. Mex. Hist. Nat., 157-175.

Jost, L. (2006). Entropy and diversity. Oikos, 113, 363-375.

Kaandorp, J. A. (1999). Morphological analysis of growth forms of branching marine sessile organisms along environmental gradients. Mar. Biol., 134, 295-306.

LaJeunesse, T. C. (2002). Diversity and community structure of symbiotic dinoflagellates from Caribbean coral reefs. Mar. Biol., 141, 387-400.

Lawrence, J. M. \& Agatsuma, Y. (2013). Tripneustes. In J. M. Lawrence (Ed.), Sea Urchins: Biology and Ecology (pp. 491502). United Kingdom, London: Elsevier. https://doi.org/10.1016/ B978-0-12-396491-5.00032-0

Lessios, H. A. (1998). Shallow water echinoids of Cayos Cochinos, Honduras. Rev. Biol. Trop., 46(Suppl. 4), 95-101.

McClanahan, T. R. \& Muthiga, N. A. (2013). Echinometra. In J. M. Lawrence (Ed.), Sea Urchins: Biology and Ecology (pp. 337354). United Kingdom, London: Elsevier. https://doi.org/10.1016/ B978-0-12-396491-5.00023-X

Moreno, C. E., Barragán, F., Pineda, E. \& Pavón, N. P. (2011). Reanálisis de la diversidad alfa: alternativas para interpretar y comparar información sobre comunidades ecológicas. Rev. Mex. Biodiv., 82(4), 1249-1261.
Mumby, P. J., Broad, K., Brumbaugh, D. R., Dahlgre, C., Harborne, A. R., Hastings, A. \&... Sanchirico, J. N. (2008). Coral reef habitats as surrogates of species, ecological functions, and ecosystem services. Conserv. Biol., 22, 941-951.

Pawson, D. L., Vance, D. J., Messing, C. G., Solís-Marín, F. A. \& Mah, C. L. (2009). Echinodermata of the Gulf of Mexico. In D. L. Felder \& D. K. Camp (Eds.), Gulf of MexicoOrigins, and Biota, Biodiversity (pp. 1117-1204). Texas: Texas A\&M University Press, College Station.

Pérez-España, H., Ávila-Gutiérrez, P. S., Melo-Merino, S. M., BerumenSolórzano, P. \& Flores-Arévalo, R. R. (2015). Patrones interanuales e interarrecifales de las comunidades de peces, corales y equinodermos en el Sistema Arrecifal Veracruzano. En A. Granados-Barba, L. Ortiz-Lozano, D. Salas-Monreal \& C. González-Gándara (Eds.), Aportes al conocimiento del Sistema Arrecifal Veracruzano: hacia el Corredor Arrecifal del Suroeste del Golfo de México (pp. 157-178). México: Universidad Autónoma de Campeche.

Sebens, K. P. \& Miles, J. S. (1988). Sweeper tentacles in a gorgonian octocoral: morphological modifications for interference competition. Biol. Bull., 175, 378-387.

SEMARNAT \& CONANP. (2014). Área de protección de flora y fauna, Sistema Arrecifal Lobos-Tuxpan. Programa de Manejo. 1ra. edición. 
México. Recuperado el 9 de agosto, 2017, disponible en http://www. conanp.gob.mx/datos_abiertos/ DGCD/125.pdf

Silva, F. J., Gómez, P. B., Santana, C. E., Silva, M. J., Lima, P. E., Santos, A. M. M. \& Pérez, D. C. (2015). Growth of the tropical zoanthid Palythoa caribaeorum (Cnidaria: Anthozoa) on reefs in northeastern Brazil. An. Acad. Bras. Cienc., 87(2), 1-12. http://dx.doi.org/10.1590/00013765201520140475.

Solís-Marín, F. A., Laguarda-Figueras, A. \& Gordillo-Hernández, M. A. (2007). Estudio taxonómico de los equinodermos del Parque Nacional Sistema Arrecifal Veracruzano. En A. Granados-Barba, L. AbarcaArenas \& J. M. Vargas-Hernández (Eds.), Investigaciones cientificas en el Sistema Arrecifal Veracruzano (pp. 73-100). México: Universidad Autónoma de Campeche.

Solís-Marín, F. A., Honey-Escandón, M. B. I., Herrero-Perezrul, M. D., Benditez-Villalobos, F., Díaz-Martínez, J. P., Buitrón-Sánchez, B. E., Pelleiro-Nayar, J. S. \& DuránGonzález, A. (2013). The Echinoderms of Mexico: Biodiversity, Distribution and Current State of Knowledge. In J. J. Alvarado \& F. A. Solís-Marín (Eds.), Echinoderm Research and Diversity in Latin America (pp. 11-65). Berlin, Heidelberg: Springer-Verlag.

Solís-Marín, F. A., Laguarda-Figueras, A. \& Honey-Escandón, M. (2014).
Biodiversidad de equinodermos (Echinodermata) en México. Rev. Mex. Biodiv., 85, 441-449.

StatSoft. (2005). Statistica (data analysis software system), version 7.1. EE.UU.: Tulsa, OK, StatSoft, Inc.

Stropes, A. (2003). Linckia guildingii (On-line), Animal Diversity Web. Recuperado el 8 de julio, 2017, disponible en http://animaldiversity. org/accounts/Linckia_guildingii/

Ter Braak, C. J. F. \& Smilauer, P. (2003). Program CANOCO, version 4.52. Biometris-quantitative methods in the life and earth sciences. The Netherlands, Wageningen: Plant Research International, Wageningen University and Research Centre.

Tunnell, J. W. Jr. (2010). Distribución de los arrecifes. En J. W. Jr. Tunnell, E. A. Chávez \& K. Withers (Eds.), Arrecifes Coralinos del Sur del Golfo de México (pp. 17-29). México: Instituto Politécnico Nacional.

Vázquez-Bader, A. R., Laguarda-Figueras, A., Gracia-Gasca, A., SolísMarín, F. A., Celaya-Hernández, E. V. \& Durán-González, A. (2008). Seasonal changes in the density and species composition of the epifaunal echinoderms recorded from the Southwester Gulf of Mexico. Rev. Biol. Trop., 56(3), 297-310.

Walther, B. A. \& Moore, J. L. (2005). The concepts of bias, precision and accuracy, and their use in testing the performance of species richness estimators, with a literature review of estimator performance. Ecography, 28, 815-829. 